\title{
Antifungal Agent F901318
}

National Cancer Institute

\section{Source}

National Cancer Institute. Antifungal Agent F901318. NCI Thesaurus. Code C116324.

A systemic antifungal agent that can potentially be used in the treatment of systemic fungal infections. 